\title{
Observation of the hyperfine spectrum of antihydrogen
}

M. Ahmadi ${ }^{1}$, B. X. R. Alves ${ }^{2}$, C. J. Baker ${ }^{3}$, W. Bertsche ${ }^{4,5}$, E. Butler ${ }^{6}$, A. Capra ${ }^{7}$, C. Carruth ${ }^{8}$, C. L. Cesar ${ }^{9}$, M. Charlton $^{3}$, S. Cohen $^{10}$, R. Collister ${ }^{7}$, S. Eriksson ${ }^{3}$, A. Evans ${ }^{11}$, N. Evetts ${ }^{12}$, J. Fajans ${ }^{8}$, T. Friesen ${ }^{2}$, M. C. Fujiwara ${ }^{7}$, D. R. Gill ${ }^{7}$, A. Gutierrez ${ }^{12,13}$, J. S. Hangst ${ }^{2}$, W. N. Hardy ${ }^{12}$, M. E. Hayden ${ }^{14}$, C. A. Isaac ${ }^{3}$, A. Ishida ${ }^{15}$, M. A. Johnson ${ }^{4,5}$, S. A. Jones ${ }^{3}$, S. Jonsell ${ }^{16}$, L. Kurchaninov ${ }^{7}$, N. Madsen ${ }^{3}$, M. Mathers ${ }^{17}$, D. Maxwell ${ }^{3}$, J. T. K. McKenna ${ }^{7}$, S. Menary ${ }^{17}$, J. M. Michan ${ }^{7,18}$, T. Momose ${ }^{12}$, J. J. Munich ${ }^{14}$, P. Nolan ${ }^{1}$, K. Olchanski ${ }^{7}$, A. Olin ${ }^{7,19}$, P. Pusa ${ }^{1}$, C. Ø. Rasmussen ${ }^{2}$, F. Robicheaux ${ }^{20}$, R. L. Sacramento ${ }^{9}$, M. Sameed ${ }^{3}$, E. Sarid ${ }^{21}$, D. M. Silveira ${ }^{9}$, S. Stracka ${ }^{7}, 22$, G. Stutter ${ }^{2}$, C. So ${ }^{11}$, T. D. Tharp ${ }^{23}$, J. E. Thompson ${ }^{17}$, R. I. Thompson ${ }^{11}$, D. P. van der Werf ${ }^{3,24} \&$ J. S. Wurtele ${ }^{8}$

The observation of hyperfine structure in atomic hydrogen by Rabi and co-workers ${ }^{1-3}$ and the measurement ${ }^{4}$ of the zero-field groundstate splitting at the level of seven parts in $10^{13}$ are important achievements of mid-twentieth-century physics. The work that led to these achievements also provided the first evidence for the anomalous magnetic moment of the electron ${ }^{5-8}$, inspired Schwinger's relativistic theory of quantum electrodynamics ${ }^{9,10}$ and gave rise to the hydrogen maser ${ }^{11}$, which is a critical component of modern navigation, geo-positioning and very-long-baseline interferometry systems. Research at the Antiproton Decelerator at CERN by the ALPHA collaboration extends these enquiries into the antimatter sector. Recently, tools have been developed that enable studies of the hyperfine structure of antihydrogen ${ }^{12}$ - the antimatter counterpart of hydrogen. The goal of such studies is to search for any differences that might exist between this archetypal pair of atoms, and thereby to test the fundamental principles on which quantum field theory is constructed. Magnetic trapping of antihydrogen atoms ${ }^{13,14}$ provides a means of studying them by combining electromagnetic interaction with detection techniques that are unique to antimatter ${ }^{12,15}$. Here we report the results of a microwave spectroscopy experiment in which we probe the response of antihydrogen over a controlled range of frequencies. The data reveal clear and distinct signatures of two allowed transitions, from which we obtain a direct, magneticfield-independent measurement of the hyperfine splitting. From a set of trials involving 194 detected atoms, we determine a splitting of $1,420.4 \pm 0.5$ megahertz, consistent with expectations for atomic hydrogen at the level of four parts in $10^{4}$. This observation of the detailed behaviour of a quantum transition in an atom of antihydrogen exemplifies tests of fundamental symmetries such as charge-parity-time in antimatter, and the techniques developed here will enable more-precise such tests.

In an earlier experiment ${ }^{12}$ using the original ALPHA apparatus ${ }^{16}$, we demonstrated microwave-induced spin flips in trapped antihydrogen. The current work was carried out using the second-generation ALPHA-2 device (Fig. 1), operating at the CERN Antiproton Decelerator ${ }^{17}$. Unlike their matter counterparts, antihydrogen atoms must be synthesized ${ }^{18}$ by merging cold plasmas of antiprotons and positrons in specially configured Penning-Malmberg traps. In the
ALPHA-2 device, we typically mix 90,000 antiprotons, slowed and captured from the Antiproton Decelerator, with 1.6 million positrons from a Surko-type accumulator ${ }^{19}$ to produce about 25,000 antihydrogen atoms. These numbers are monitored in daily baseline measurements that involve ejecting particles onto a multichannel plate detector.

Of the produced antihydrogen atoms, only a few will have low enough kinetic energies ( $0.54 \mathrm{~K}$ in temperature units) to be trapped in our superconducting, multipolar, magnetic-minimum trap. The current state-of-the-art is that about 20 atoms can be trapped from a single mixing sequence, and we have accumulated up to 74 atoms by repetitive mixing (M.A. et al., submitted). A single mixing and capture sequence takes approximately $4 \mathrm{~min}$, the bulk of which is used for preparation of plasmas of appropriate temperature, size and density (M.A. et al., submitted). The actual mixing process takes less than $1 \mathrm{~s}$. The trapped antimatter atoms can survive for at least $1,000 \mathrm{~s}$ in the cryo-pumped ultrahigh vacuum of ALPHA-2.

Referring to Fig. 1, the antiproton and positron plasmas are merged in the central Penning trap (yellow electrodes) to produce antihydrogen. An external solenoid magnet provides a uniform 1-T field for the Penning trap. The production region is near the centre of the magnetic-minimum trap, which comprises an octupole coil for transverse confinement of neutral anti-atoms and five short solenoids ('mirror coils') that can shape the axial trapping well. The trapping volume is cylindrical, with a diameter of $44.35 \mathrm{~mm}$ and length of $280 \mathrm{~mm}$. For the current experiment, only the outer two mirror coils are used to create the axial well.

Antihydrogen atoms that leave the trap and annihilate on the electrodes of the Penning trap are registered by the ALPHA-2 annihilation detector ${ }^{20}$. This three-layer silicon vertex detector that surrounds the trapping volume (Fig. 1) determines the vertex position of the antiproton annihilation. The amount of trapped antihydrogen can be determined destructively at any time by intentionally ramping down the trapping magnets to release anti-atoms, while monitoring their annihilations. The dominant background in our experiment comes from cosmic rays, which trigger the detector at an average rate of $10.02 \pm 0.02 \mathrm{~s}^{-1}$ (all errors herein are one standard deviation).

To distinguish antiproton annihilations from cosmic rays, we use extended versions of our previously developed methods of

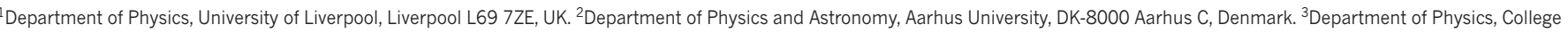

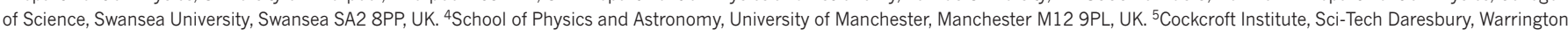

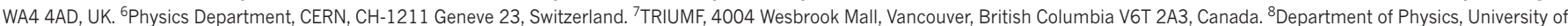

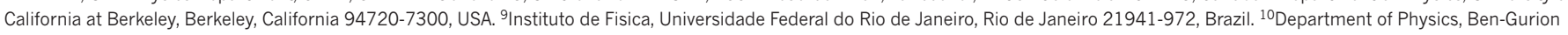
University of the Negev, Beer-Sheva 84105, Israel. ${ }^{11}$ Department of Physics and Astronomy, University of Calgary, Calgary, Alberta T2N 1N4, Canada. ${ }^{12}$ Department of Physics and Astronomy, University of British Columbia, Vancouver, British Columbia V6T 1Z1, Canada. ${ }^{13}$ Department of Medical Physics and Biomedical Engineering, University College London, London WC1E 6BT, UK. ${ }^{14}$ Department of Physics, Simon Fraser University, Burnaby, British Columbia V5A 1S6, Canada. ${ }^{15}$ Department of Physics, The University of Tokyo, 7-3-1 Hongo, Bunkyo, Tokyo 113-0033, Japan.

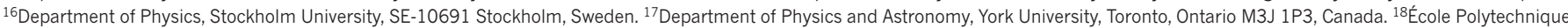
Fédérale de Lausanne (EPFL), Swiss Plasma Center (SPC), Lausanne CH-1015, Switzerland. ${ }^{19}$ Department of Physics and Astronomy, University of Victoria, Victoria, British Columbia V8P 5C2, Canada. ${ }^{20}$ Department of Physics and Astronomy, Purdue University. West Lafayette, Indiana 47907, USA. ${ }^{21}$ Soreq NRC, Yavne 81800, Israel. ${ }^{22}$ Universita di Pisa and Sezione INFN di Pisa, Largo Pontecorvo 3, 56127 Pisa, Italy. ${ }^{23}$ Physics Department, Marquette University, PO Box 1881, Milwaukee, Wisconsin 53201-1881, USA. ${ }^{24}$ IRFU, CEA/Saclay, F-91191, Gif-sur-Yvette Cedex, France. 


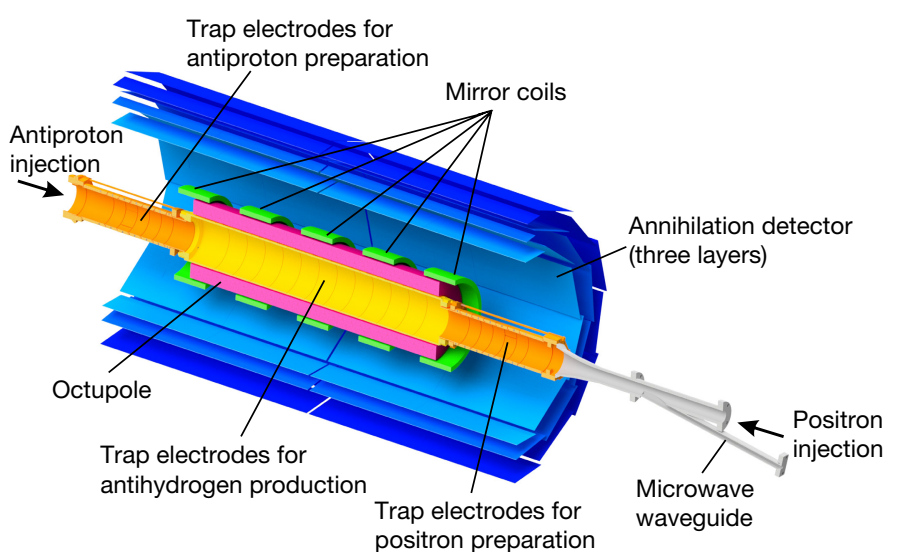

Figure 1 | The ALPHA-2 central apparatus. A cut-away schematic of the antihydrogen production and trapping region of ALPHA-2 is shown. For clarity, the vacuum wall and the cryostat for the superconducting magnets are not shown. Antiproton and positron plasmas are prepared on either side of the production region before being mixed to form antihydrogen at the centre of the minimum- $B$ trap. All of the components shown are immersed in a uniform, 1-T, axial magnetic field, which is provided by an external solenoid (not illustrated).

multivariate analysis ${ }^{12}$ (Methods). The multivariate analysis used for the current experiment yields a cosmic ray background rate of $0.00559 \pm 0.00051 \mathrm{~s}^{-1}$ and an overall detection efficiency for annihilations of $0.596 \pm 0.002$.

Figure 2 depicts the expected energy levels of ground-state antihydrogen in a magnetic field $\boldsymbol{B}$. Atoms in either of the two levels labelled $|c\rangle$ and $|d\rangle$ have energies that increase with field strength $B$ and can thus be trapped in a minimum- $B$ configuration. The other two states, labelled $|a\rangle$ and $|b\rangle$, are expelled from the trap. The essential idea of the experiment is to use microwaves at about $29 \mathrm{GHz}$ to resonantly drive transitions from trapped to un-trapped states as anti-atoms traverse the bottom of the magnetic potential well. At $1 \mathrm{~T}$, the $|c\rangle \rightarrow|b\rangle$ and $|d\rangle \rightarrow|a\rangle$ transitions correspond to positron spin flips. Calculations for hydrogen (Fig. 3, inset) show that the inhomogeneous magnetic field produces asymmetric line shapes, with sharply defined onsets corresponding to the resonant frequency of either transition at the field minimum. The frequency difference between the two onsets represents the ground-state hyperfine splitting and is independent of the field strength and the number of anti-atoms that are trapped. It is this splitting that we seek to measure in antihydrogen and compare to that in hydrogen ${ }^{9,10}$.

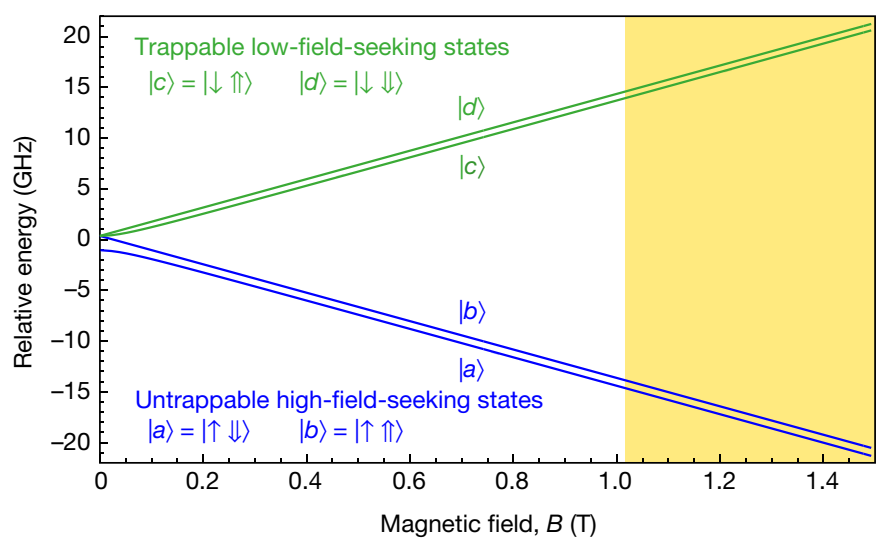

Figure 2 | Ground-state hyperfine energy levels. The energy levels are calculated assuming they are identical to those of hydrogen. The ket notation indicates the positron spin (left; $\downarrow$ or $\uparrow$ ) and antiproton spin (right; $\Downarrow$ or $\Uparrow$ ) states in the high-field limit. The shaded region illustrates part of the range of fields in the ALPHA-2 antihydrogen trap, with the minimum at $1.03 \mathrm{~T}$. The full field map is shown in Fig. 3.

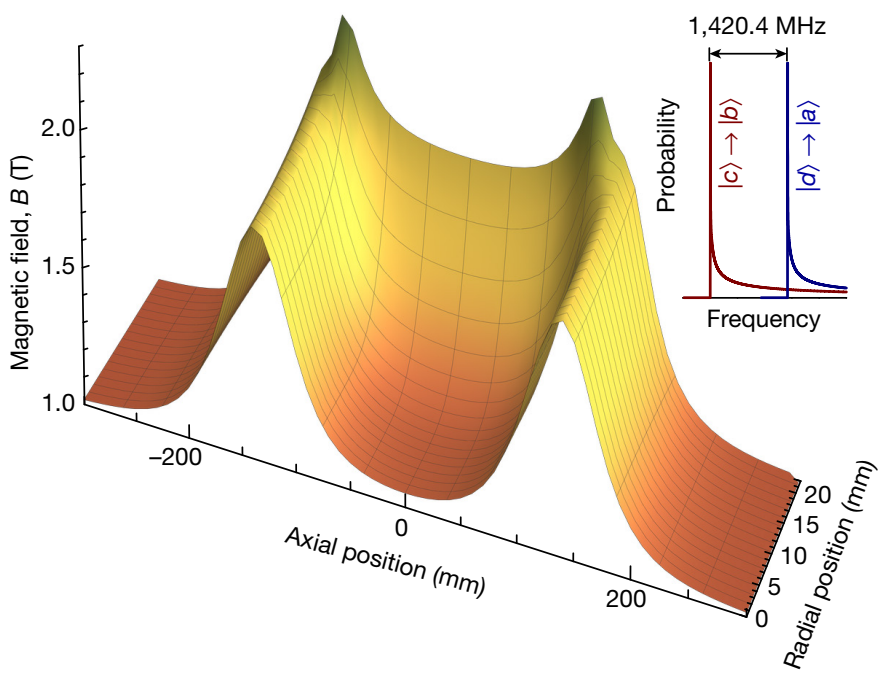

Figure 3 | Magnetic field strength. The scalar magnitude of the total magnetic field in the atom trap is plotted versus position. The radial position is measured from the symmetry axis of the trap, and the axial origin is the centre of the atom trap, defined by the outermost mirror coils. Inset, the probability of an atom being resonant at a given frequency, calculated for hydrogen; the two low-frequency onsets are separated by the ground-state splitting.

In Fig. 3 we show the magnetic field strength in ALPHA-2 as a function of position in the trapping volume. The volume of interest is at the centre of the trap, where fields, in the vicinity of the minimum, are about $1 \mathrm{~T}$. Microwaves enter the apparatus through a purpose-built vacuum feed-through and are directed to the trapping volume using a waveguide (Fig. 1). We use an Agilent 8257D PSG frequency synthesizer and a Miteq AMF-4B amplifier to generate the microwave power. We injected $160 \mathrm{~mW}$ and $320 \mathrm{~mW}$ at the lower and upper transitions, respectively (see the discussion below); both are measured at the feed-through.

The experimental procedure involves producing and trapping antihydrogen atoms, removing any residual charged particles from the trap using pulsed electric fields, and then introducing microwaves into the trapping volume. The microwave frequency is stepped up in $300-\mathrm{kHz}$ increments, starting from below the expected onset frequency of the $|c\rangle \rightarrow|b\rangle$ transition. The trapped antihydrogen atoms are exposed to microwave fields at each frequency for $4 \mathrm{~s}$. After the first 16 steps, the starting frequency is incremented by $+1,420.4 \mathrm{MHz}$ and the process is repeated to scan through the onset of the $|d\rangle \rightarrow|a\rangle$ transition. The silicon vertex detector continuously monitors for the annihilation of antihydrogen atoms that are ejected following a resonant spin flip. The total illumination cycle is $2 \times 64 \mathrm{~s}$ ( 16 points spanning the onset of each transition), after which the trap is de-energized in $1.5 \mathrm{~s}$, releasing any remaining antihydrogen.

For the dataset presented here, we repeated this measurement sequence 22 times over a three-day period. A combination of single and double mixing sequences was used, achieving an average trapping rate of about 14 atoms per trial. Each day, before data acquisition, the external solenoid field was reset and the minimum field strength at the centre of the magnetic trap was determined by measuring the electron cyclotron resonance frequency of an electron plasma ${ }^{21}$. The precision of this measurement is estimated to be $\pm 0.3 \mathrm{mT}$ (equivalent to $8.4 \mathrm{MHz}$ in electron cyclotron resonance frequency).

The results of the 22 measurement trials are plotted in Fig. 4. Cosmic background contributes $0.492 \pm 0.045$ events to each 4 -s measurement bin. The sums of each day's trials are combined by aligning the maxima of the lower $(|c\rangle \rightarrow|b\rangle)$ transition, to account for day-to-day variations in magnetic field. The responses observed should not be directly compared to traditional spectral lines or to the calculated distributions in the inset to Fig. 3, because the detailed shape is strongly influenced 


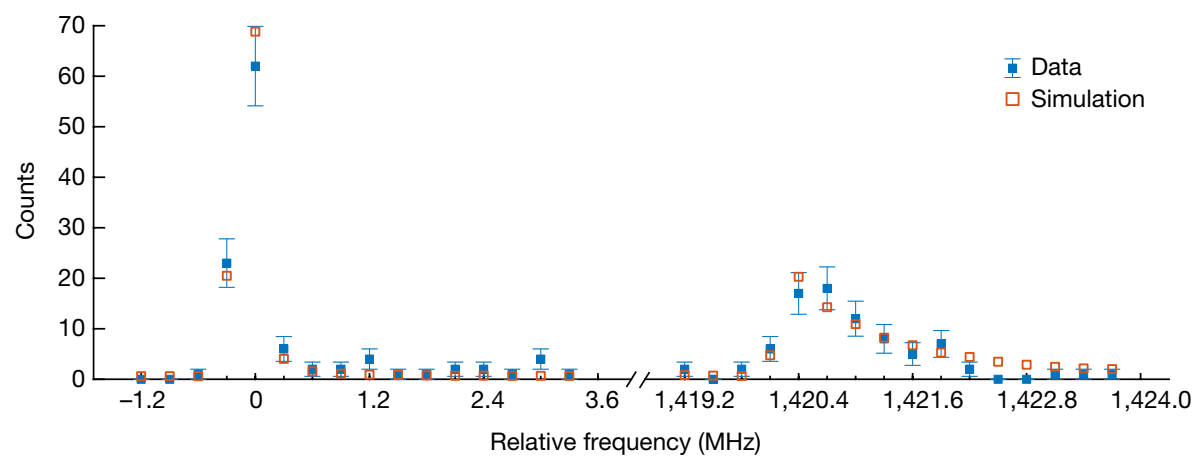

Figure 4 | Data and simulation. The number of detected antihydrogen annihilation events (filled blue squares), summed (see the text for details) over 22 trials, is plotted as a function of frequency. Note the discontinuous abscissa. The error bars represent counting statistics only. The expectations from the simulation for hydrogen in the trap are also

by the rate at which the trap is depopulated as atoms undergo spin-flip transitions.

Figure 4 reveals a qualitative difference between the shapes of the responses for the two transitions. The lower transition features a low-frequency onset and a narrow width; most of the anti-atoms are removed in the first two resonant bins. The more gradual onset and broader width of the upper transition are attributed to a lower amplitude of the in situ microwave magnetic field (see simulation results below). As illustrated in Fig. 1, the Penning-trap electrode stack in ALPHA-2 represents a complicated boundary surface for the injected electromagnetic radiation. It is unsurprising that disparate mixtures of standing and travelling waves are established at different frequencies. We can measure the strengths of the microwave electric fields in the vicinity of the two transitions by studying electron cyclotron resonance heating of electron plasmas stored in the trap centre ${ }^{21}$. These measurements lead to the conclusion that, for a given injected power, the microwave electric field strength at the centre of the trap is about seven times stronger at the lower transition frequency than at the higher one. However, we do not know the precise in situ relationship between the microwave electric and magnetic fields; and it is the latter that drives transitions between hyperfine levels. To partially compensate for this imbalance, we injected twice as much power at the upper transition, relative to the lower transition. This reduces the ratio of microwave-field amplitudes at the two transitions from seven to five. Our ability to further balance these amplitudes is currently limited by adverse thermal effects in the cryogenic, ultrahigh-vacuum environment.

Also shown in Fig. 4 are the results of a simulation (Methods) of the expected behaviour of hydrogen atoms in the magnetic environment of our trap. The inputs to this simulation include the trapping magnetic fields that are calculated from measured currents, the amplitudes of the microwave magnetic field that are inferred via electron cyclotron resonance and assuming plane-wave propagation in vacuum, the starting frequency for the microwave scan, the energy distribution of the trapped atoms, and the expected temporal magnetic field fluctuations, which are based on current-transformer monitoring of the currents in the trapping magnets. The simulation result is scaled to give the same total number of events as the experiment, integrated over both transitions (194 detected events). The simulation explicitly accounts for the removal of atoms from the trap as spin-flip events occur.

As described above, our experimental protocol was designed to determine the difference between the onset frequencies for the two transitions, profiting from the expected sharp increase in signal associated with resonance at the magnetic-field minimum. The slower increase that was observed in the $|d\rangle \rightarrow|a\rangle$ transition complicates the determination of the frequency splitting. It is tempting to extract a 'best' value for the hyperfine splitting by fitting the hydrogen simulation to the experimental data, particularly because the simulation reproduces the form of the data rather well. Given the fundamental nature of the shown (open red squares). The simulation results are scaled to match the total number (194) of observed events and are aligned to match the onset of the lower transition. The simulation includes the effect of measured fluctuations in the currents in the trapping magnets.

quantity that is to be extracted, however, we defer any such interpretation until more detailed systematic studies can be performed.

We determine the splitting from Fig. 4 to be $1,420.4 \pm 0.5 \mathrm{MHz}$, which reflects the difference between the low-frequency onsets of the two lines. The uncertainty includes contributions from drifts in the magnetic field that were observed during the scan $(0.3 \mathrm{MHz})$, the procedure used for combining the data from separate days $(0.3 \mathrm{MHz})$, and the determination of the onset frequencies of the two lines $(0.3 \mathrm{MHz})$. This is the only available direct measurement of this fundamental quantity. The precision of our technique can be improved by reducing the step size in the frequency scan, by balancing the microwave power at the two transitions and by more precise characterization and stabilization of the magnetic fields in the atom trap. There are also plans to measure the same quantity in zero magnetic field using a beam of antihydrogen ${ }^{22}$.

The release of the antihydrogen atoms that remain in the trap after the two transitions have been illuminated yielded 9 detected events for the 22 trials. A different multivariate analysis is used for this determination (Methods); the overall efficiency is 0.726 and we expect 1.3 total background events for the 22 trials. We conclude that about $96 \%$ of the trapped anti-atoms were removed as a result of a spin flip. Independent measurements using only the lower transition indicate that a microwave power sufficient to remove all of the trapped atoms with a 1-s time constant was injected without adverse thermal effects in the cryogenic, ultrahigh-vacuum system. In addition to being a useful diagnostic for optimizing antihydrogen trapping, the ability to selectively control the populations of the trapped quantum states will be useful for future microwave and optical spectroscopy of trapped antihydrogen.

The work described exemplifies a new approach in antimatter physics: the observation of spectral line shapes in antihydrogen. The ability to make a controlled frequency scan over an expected quantum mechanical transition in an atom of antimatter points the way to more precise tests of fundamental symmetries with antihydrogen. Charge-parity-time invariance implies that the detailed shapes-not just the resonance frequencies-of spectral lines for hydrogen and antihydrogen in the same environment must be identical. We will soon be able to use such precise measurements to subject antihydrogen to previously unobtainable scrutiny.

Also of interest is the nuclear magnetic resonance (NMR)-type transition between the $|c\rangle$ and $|d\rangle$ states, which corresponds to an antiproton spin flip. Recent advances in trapping efficiency (M.A. et al., submitted) bode well for the feasibility of observing this transition in trapped antihydrogen. The absolute energy scales for the positron and antiproton spin-flip transitions in ALPHA-2 are respectively five and eight orders of magnitude smaller than that of the laser transition that was recently observed ${ }^{15}$. In addition to probing different interactions in the antihydrogen Hamiltonian, these energy scales offer very high sensitivity to potential new physics ${ }^{23}$. 
Online Content Methods, along with any additional Extended Data display items and Source Data, are available in the online version of the paper; references unique to these sections appear only in the online paper.

\section{Received 27 May; accepted 30 June 2017.}

1. Rabi, I. I., Kellogg, J. M. B. \& Zacharias, J. R. The magnetic moment of the proton. Phys. Rev. 46, 157-163 (1934).

2. Rabi, I. I., Kellogg, J. M. B. \& Zacharias, J. R. The magnetic moment of the deuteron. Phys. Rev. 46, 163-165 (1934).

3. Kellogg, J. M. B., Rabi, I. I. \& Zacharias, J. R. The gyromagnetic properties of the hydrogens. Phys. Rev. 50, 472-481 (1936).

4. Petit, P., Desaintfuscien, M. \& Audoin, C. Temperature dependence of the hydrogen maser wall shift in the temperature range 295-395 K. Metrologia 16, 7-14 (1980)

5. Nafe, J. E., Nelson, E. B. \& Rabi, I. I. The hyperfine structure of atomic hydrogen and deuterium. Phys. Rev. 71, 914-915 (1947).

6. Nafe, J. E. \& Nelson, E. B. The hyperfine structure of hydrogen and deuterium. Phys. Rev. 73, 718-728 (1948).

7. Nelson, E. B. \& Nafe, J. E. The hyperfine structure of tritium. Phys. Rev. 75, 1194-1198 (1949).

8. Nelson, E. B. \& Nafe, J. E. A comparison of the $g$ value of the electron in hydrogen with that in deuterium. Phys. Rev. 76, 1858-1860 (1949).

9. Schwinger, J. On quantum-electrodynamics and the magnetic moment of the electron. Phys. Rev. 73, 416-417 (1948).

10. Schwinger, J. Quantum electrodynamics. III. The electromagnetic properties of the electron-radiative corrections to scattering. Phys. Rev. 76, 790-817 (1949).

11. Goldenberg, H. M., Kleppner, D. \& Ramsey, N. F. Atomic hydrogen maser. Phys. Rev. Lett. 5, 361-362 (1960).

12. Amole, C. et al. Resonant quantum transitions in trapped antihydrogen atoms. Nature 483, 439-443 (2012).

13. Andresen, G. B. et al. Trapped antihydrogen. Nature 468, 673-676 (2010).

14. Andresen, G. B. et al. Confinement of antihydrogen for 1,000 seconds. Nat. Phys. 7, 558-564 (2011).

15. Ahmadi, M. et al. Observation of the 1 S-2S transition in trapped antihydrogen. Nature 541, 506-510 (2017)

16. Amole, C. et al. The ALPHA antihydrogen trapping apparatus. Nucl. Instrum Methods A 735, 319-340 (2014).

17. Maury, S. The antiproton decelerator: AD. Hyperfine Interact. 109, 43-52 (1997)

18. Amoretti, M. et al. Production and detection of cold antihydrogen atoms. Nature 419, 456-459 (2002).

19. Murphy, T. J. \& Surko, C. M. Positron trapping in an electrostatic well by inelastic collisions with nitrogen molecules. Phys. Rev. A 46, 5696-5705 (1992).

20. Amole, C. et al. Silicon vertex detector upgrade in the ALPHA experiment. Nucl. Instrum. Methods A 732, 134-136 (2013).

21. Amole, C. et al. In situ electromagnetic field diagnostics with an electron plasma in a Penning-Malmberg trap. New J. Phys. 16, 013037 (2014).
22. Kuroda, N. et al. A source of antihydrogen for in-flight hyperfine spectroscopy. Nat. Commun. 5, 3089 (2014).

23. Kostelecký, V. A. \& Vargas, A. J. Lorentz and CPT tests with hydrogen, antihydrogen, and related systems. Phys. Rev. D 92, 056002 (2015).

Acknowledgements All authors are members of the ALPHA Collaboration. This work was supported by: the European Research Council through its Advanced Grant programme (J.S.H.); CNPq, FAPERJ and RENAFAE (Brazil); NSERC, NRC/TRIUMF, EHPDS/EHDRS and FQRNT (Canada); FNU (NICE Centre) and Carlsberg Foundation (Denmark); JSPS Postdoctoral Fellowships for Research Abroad (Japan); ISF (Israel); STFC, EPSRC, the Royal Society and the Leverhulme Trust (UK); DOE and NSF (USA); and VR (Sweden). We are grateful for the efforts of the CERN Antiproton Decelerator team. We thank J. Tonoli (CERN) and his staff for help with machining work. We thank the staff of the Superconducting Magnet Division at Brookhaven National Laboratory for collaboration and fabrication of the trapping magnets. We thank C. Marshall (TRIUMF) for his work on the ALPHA-2 cryostat. We thank F. Besenbacher (Aarhus) for support in procuring the ALPHA-2 external solenoid.

Author Contributions This experiment was based on data collected using the ALPHA-2 antihydrogen trapping apparatus, which was designed and constructed by the ALPHA Collaboration using methods developed by the entire collaboration. The entire collaboration participated in the operation of the apparatus and the data-taking activities. The experimental protocol was conceived by M.E.H. and T.F. The microwave hardware was implemented by T.F., W.N.H., A.I., J.J.M. and M.E.H. Detailed analysis of the antiproton annihilation detector data was done by J.T.K.M. and A.O., using methods introduced to ALPHA by S.S. The manuscript was written by J.S.H., T.F., M.E.H., N.M., J.T.K.M. and F.R., and was then edited and improved by the entire collaboration.

Author Information Reprints and permissions information is available at www.nature.com/reprints. The authors declare no competing financial interests. Readers are welcome to comment on the online version of the paper. Publisher's note: Springer Nature remains neutral with regard to jurisdictional claims in published maps and institutional affiliations. Correspondence and requests for materials should be addressed to J.S.H. (jeffrey.hangst@cern.ch), T.F. (Tim.Friesen@cern.ch) or M.E.H. (mhayden@sfu.ca).

Reviewer Information Nature thanks R. Pohl and the other anonymous reviewer(s) for their contribution to the peer review of this work.

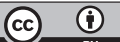

This work is licensed under a Creative Commons Attribution 4.0 International (CC BY 4.0) licence. The images or other third party material in this article are included in the article's Creative Commons licence, unless indicated otherwise in the credit line; if the material is not included under the Creative Commons licence, users will need to obtain permission from the licence holder to reproduce the material. To view a copy of this licence, visit http://creativecommons.org/licenses/by/4.0/. 


\section{METHODS}

Simulation of the microwave-atom interaction. Our simulation of the microwave flip process uses quantum and classical ideas. The motion of antihydrogen through the trap is determined by solving the classical equations of motion using a fourth-order symplectic integrator. The force on the atom arises from the spatially dependent magnetic field. Because the magnetic fields are always large, the state of the antihydrogen is conserved unless the resonance condition is satisfied. While trapped, the atoms are in either the $|c\rangle$ or $|d\rangle$ states (the low-field-seeking states). These states have a magnetic moment that is approximately equal to that of the positron, which leads to a potential energy of $\mathrm{PE} \approx \mu \mathrm{B} / 2$, where $\mu=g \mu_{\mathrm{B}}$. Here, $\mu_{\mathrm{B}}$ is the Bohr magneton and $g$ is the spin $g$-factor. The force is obtained by numerically computing the gradient of the magnitude of the magnetic field.

The energies of the hyperfine states are solved using an effective Hamiltonian with the hyperfine splitting and the magnetic moments of hydrogen as inputs. A quadratic interpolation of the energies at three consecutive time steps is used to determine whether the microwave resonance condition is met. Where in space this condition is met depends on the currents in the various magnets and on the microwave frequency. If the resonance condition occurs during the step, then the Landau-Zener approximation is used to obtain a spin-flip probability. The time derivative of the energy separation is calculated from the quadratic interpolation. The matrix element $V$ that couples the $|c\rangle$ and $|b\rangle$ or $|d\rangle$ and $|a\rangle$ states depends on the microwave magnetic field $B_{\mathrm{MW}}$ perpendicular to the static magnetic field at the position at which the antihydrogen is in resonance; it is well approximated by $V=B_{\mathrm{MW}} \mu / 4$. To estimate $B_{\mathrm{MW}}$ we use the electron-cyclotronresonance plasma-heating diagnostic discussed in the main text, which measures the microwave electric field $E_{\mathrm{MW}}$ perpendicular to the static magnetic field. We then assume $B_{\mathrm{MW}}$ is uniform and given by $B_{\mathrm{MW}}=E_{\mathrm{MW}} / c$, where $c$ is the speed of light in vacuum. (The precise relationship between $E_{\mathrm{MW}}$ and $B_{\mathrm{MW}}$ is not known because the boundary conditions imposed by the electrode stack support a complex mixture of standing and travelling wave modes. Errors from making this assumption are reduced by averaging.)

Resonance conditions are encountered in pairs as atoms pass through the centre of the trap, and we account for the possibility that spins will flip more than once. Simulations do not predict a simple exponential decay of trapped populations when microwaves are present; the rate at which atoms encounter resonance conditions and the probability that they undergo a spin flip as they pass through resonance vary with trajectory. However, as an indication of scale, a microwave intensity of $4 \mathrm{~mW} \mathrm{~cm}^{-2}$ (corresponding to $B_{\mathrm{MW}}=0.6 \mu \mathrm{T}$ and $V=h \times(4 \mathrm{kHz}$ ), where $h$ is Planck's constant) applied just above the onset of either transition will clear atoms in the corresponding state from the trap with a time constant of order $1 \mathrm{~s}$.

Multivariate analysis of detector events. Differentiation of antihydrogen annihilations and background events (primarily cosmic rays) is achieved by discerning their distinctive topologies. A multivariate analysis package is used to distinguish between these two populations ${ }^{24,25}$.

Two independent multivariate analyses were performed for this experiment: a low-background analysis for identifying annihilations during the 128-s microwave window and a high-signal-acceptance analysis to identify annihilations during the 1.5-s trap shutdown. The latter analysis has the same design as used in previous experiments ${ }^{12,16}$. The former analysis is modified to achieve a much lower background, through the addition of more variables that enhance the signal-to-background discrimination. The additional variables include: the asymmetry in hit count between the two hemispheres defined by the plane perpendicular to the event axis ${ }^{12}$ and passing through the centre of the trap; the minimum distance of closest approach of any cosmic track candidate to the reconstructed vertex; the polar angle of the vector describing the vertex position ${ }^{26}$ relative to the centre of the trap; and the average of the ratios between the axial and radial projections of the tracks that originate from the reconstructed vertex.

The signal and background data used for multivariate-analysis training, validation and testing (split equally) comprises a set of 305,706 annihilation events and 236,969 background events.

Data availability. The datasets generated and analysed during this study are available from corresponding author J.S.H. on reasonable request.

24. Narsky, I. StatPatternRecognition: a C++ package for statistical analysis of high energy physics data. Preprint at http://arxiv.org/abs/physics/0507143 (2005).

25. Narsky, I. Optimization of signal significance by bagging decision trees. Preprint at http://arxiv.org/abs/physics/0507157 (2005).

26. Amole, C. et al. Alternative method for reconstruction of antihydrogen annihilation vertices. Hyperfine Interact. 212, 101-107 (2012). 


\section{CORRECTIONS \& AMENDMENTS}

\section{ERRATUM}

doi:10.1038/nature24663

\section{Erratum:Observation of the hyperfine spectrum of antihydrogen}

M. Ahmadi, B. X. R. Alves, C. J. Baker, W. Bertsche,

E. Butler, A. Capra, C. Carruth, C. L. Cesar, M. Charlton,

S. Cohen, R. Collister, S. Eriksson, A. Evans, N. Evetts,

J. Fajans, T. Friesen, M. C. Fujiwara, D. R. Gill, A. Gutierrez,

J. S. Hangst, W. N. Hardy, M. E. Hayden, C. A. Isaac, A. Ishida,

M. A. Johnson, S. A. Jones, S. Jonsell, L. Kurchaninov,

N. Madsen, M. Mathers, D. Maxwell, J. T. K. McKenna,

S. Menary, J. M. Michan, T. Momose, J. J. Munich,

P. Nolan, K. Olchanski, A. Olin, P. Pusa, C. Ø. Rasmussen,

F. Robicheaux, R. L. Sacramento, M. Sameed, E. Sarid,

D. M. Silveira, S. Stracka, G. Stutter, C. So, T. D. Tharp,

J. E. Thompson, R. I. Thompson, D. P. van der Werf \&

J. S. Wurtele

Nature 548, 66-69 (2017); doi:10.1038/nature23446

Owing to a technical error, author J. S. Wurtele was listed incorrectly as a corresponding author instead of author J. S. Hangst in the HTML version of this Letter (the PDF version was correct). This has been corrected online. 